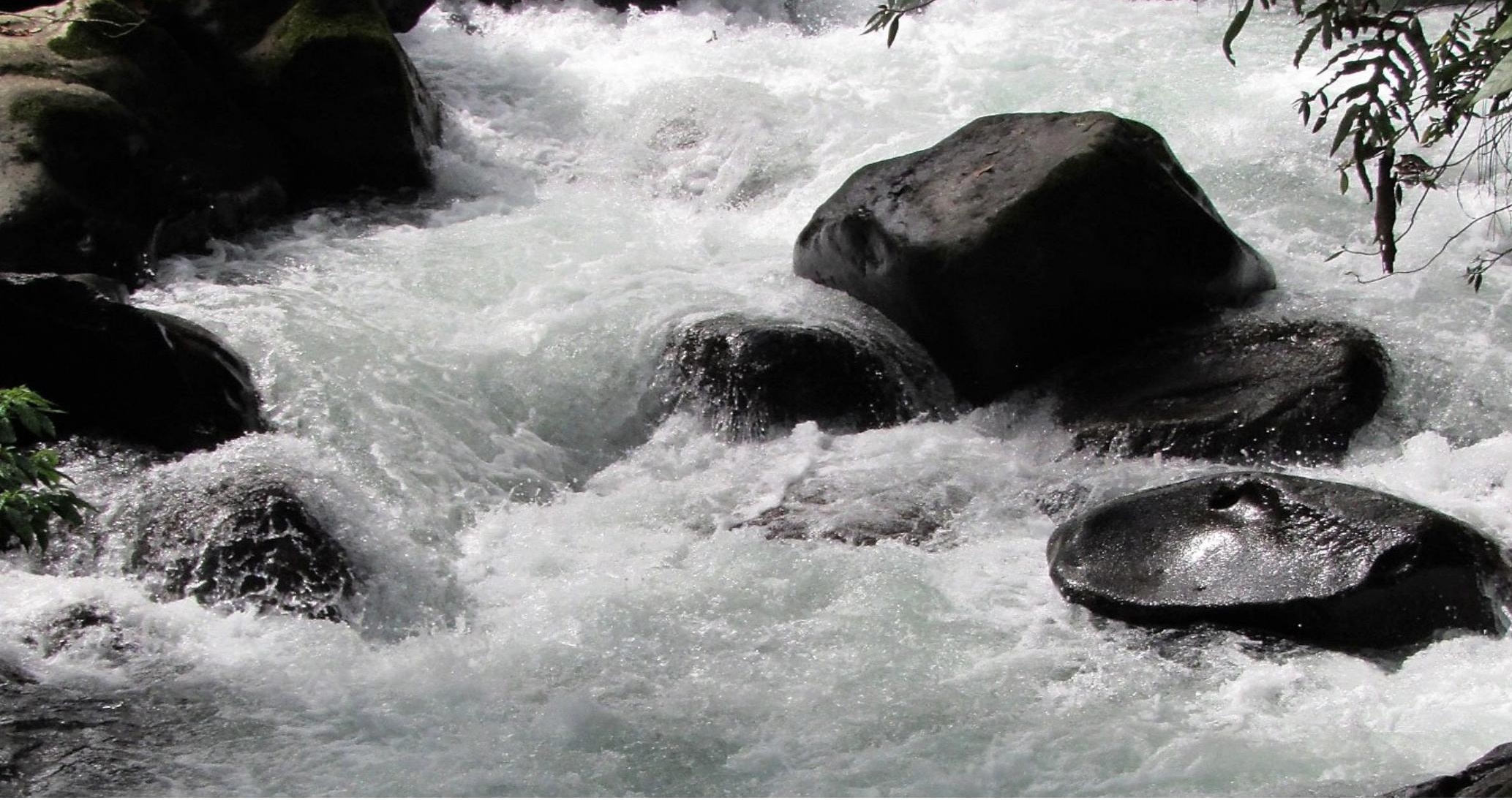

\title{
Usuarios del agua y su posible contribución a la contaminación por cadmio en fuentes naturales de la microcuenca del río Pixquiac
}

S. Menchaca1-L. M. Ríos² - L. de Medina

RESUMEN: Se analizan aspectos relativos a la degradación ambiental relacionada con las actividades de los usuarios del agua agrícola, pecuario y doméstico (factor antrópico), mismas que pueden estar contribuyendo a la contaminación del agua por cadmio en la microcuenca del río Pixquiac. La investigación muestra la importancia de evaluar tanto la calidad del recurso hídrico en cuerpos naturales, como las posibles causas antrópicas que los están contaminando, para identificar tanto el riesgo que tiene la población, como la relevancia de desarrollar estrategias de conservación de los ecosistemas acuáticos.

Palabras Clave: agua, medio ambiente, degradación, contaminación, cadmio.
ABSTRACT: This paper analyzes aspects relative to environmental degradation related to the activities of agricultural, livestock and domestic water users (anthropic factor), which may be contributing to the pollution of water by cadmium in the Pixquiac River microbasin. The research shows the importance of evaluating both the quality of water resources in natural bodies, as well as the possible anthropic causes that are contaminating them, to identify the risk for the population, and the relevance of developing strategies for the conservation of aquatic ecosystems.

Keywords: water, environment, degradation, pollution, cadmium.

1 Observatorio del Agua para el Estado de Veracruz, OABCC (Agua, Bosques, Cuencas y Costas). Centro de Ciencias de la Tierra, Universidad Veracruzana. email: socorro.menchaca@gmail.com

2 Observatorio del Agua para el Estado de Veracruz, OABCC (Agua, Bosques, Cuencas y Costas). Centro de Ciencias de la Tierra, Universidad Veracruzana. email: luis.rf94@gmail.com

3 Observatorio del Agua para el Estado de Veracruz, OABCC (Agua, Bosques, Cuencas y Costas). Centro de Ciencias de la Tierra, Universidad Veracruzana.email: loredms@hotmail.com 


\section{Introducción}

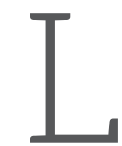

as actividades de los usuarios del agua, impactan y afectan a los servicios ambientales, mismos que se representan en las diversas funciones que desempeñan los ecosistemas. En lo específico, los servicios ambientales son beneficios intangibles, que ayudan al mantenimiento de la vida de los sistemas naturales y al bienestar de las personas y las comunidades (SEMARNAT, 2004), de ahí la relevancia de su cuidado, conservación y en su caso su restauración.

Los distintos tipos de servicios ambientales, de acuerdo con la Evaluación de los Ecosistemas del Milenio (2005), son los siguientes: los denominados de apoyo que son necesarios para la producción de materias primas, la producción de oxígeno, la formación del suelo, entre otros; los conceptualizados como de provisión que son el agua dulce, la obtención de alimentos, combustibles, fibras y los recursos energéticos, etc.; los nombrados como de regulación que incluyen el control del clima y de inundaciones, el mantenimiento de la calidad del aire, control de la erosión, la regulación de las enfermedades humanas y la purificación del agua, etc.; y los culturales, que se relacionan con el enriquecimiento espiritual, la reflexión, la recreación y las experiencias artísticas y estéticas, entre otros.

Especial atención aquí tienen los servicios ambientales conexos al agua, como son los relativos a las cuencas hidrológicas expresados en la calidad del agua superficial y subterránea, regulación de caudales, así como la productividad acuática y vegetal; y los servicios ambientales relacionados con los bosques como son la conservación de la biodiversidad, la calidad del suelo y aire, captura de carbono, microclima, retención hídrica, entre otros.

Resulta significativo que en la actualidad las fuentes naturales de agua se estén contaminando, por distintas actividades que realizan los usuarios del agua de los sectores agrícola, ganadero, acuacultura, doméstico, industrial y de servicios, etc., ya que de manera permanente dichas fuentes son impactadas y afectadas por distintos factores químicos, microbiológi- cos y/o metales pesados, entre otros,

Cabe señalar que, en el desarrollo de las actividades antrópicas, generalmente se descargan de manera periódica grandes cantidades de dichas sustancias, mismas que recibe el sistema hídrico, lo que impide que se desarrollen procesos de resiliencia en los servicios ambientales. El sistema se vuelve incapaz de soportarlo: la biodiversidad se pierde; los medios de subsistencia disminuyen; las fuentes naturales de alimentos se deterioran, se originan costos de remediación elevados (Marsily, 2003), y también se deteriora la calidad del recurso hídrico.

Cabe señalar, que la evaluación de los ecosistemas del milenio (EM), programa internacional que se concluyó en 2005, fue diseñado para la toma de decisiones y tiene como eje la generación de información científica sobre los vínculos entre las transformaciones que están sufriendo los ecosistemas y el bienestar humano.

La EM busca principalmente, contribuir a la generación de capacidades individuales e institucionales para que se evalúen de manera integral los ecosistemas, y para que de acuerdo con los resultados se establezcan estrategias, dirigidas a la conservación de los ecosistemas y sus servicios ambientales, como pueden ser los conexos al sistema hidrológico (Menchaca y Alvarado, 2011).

Cabe señalar que, la microcuenca del río Pixquiac no está exenta de las afectaciones relacionadas con el desarrollo de las actividades humanas, a pesar de que es una fuente importante de agua dulce superficial que presta servicios ambientales a Xalapa, la capital del estado de Veracruz y a los municipios que integran esa región.

Por lo anterior, el propósito general de la investigación del Observatorio del Agua para el Estado de Veracruz, OABCC (Agua, Bosques, Cuencas y Costas), es el análisis de las actividades antrópicas de los usuarios del agua de los sectores agrícola, pecuaria y doméstica que pueden contribuir a la contaminación del recurso hídrico.

La medición del factor antrópico, en el sentido anterior, no sólo permite detectar la problemática en el contexto de la degradación am- 
biental en cuanto a los impactos y afectaciones a los servicios ambientales ecosistémicos, sino también desarrollar estrategias que coadyuven al cuidado de los cuerpos de agua naturales; así como implementar mecanismos que alerten a la población sobre los riesgos a la salud por el uso de agua contaminada, principalmente a los habitantes de las áreas rurales, que en muchas ocasiones toman agua para uso humano de dichas fuentes naturales, entre otros aspectos relacionados con la gestión y manejo integrado de los recursos hídricos.

\section{Antecedentes y problemática}

En el OABCC, se han desarrollado investigaciones desde el 2011, en el contexto del impacto de las actividades antrópicas de los usuarios del agua agrícola, forestal, acuacultura, industrial y doméstica en relación a las afectaciones de los servicios ambientales ecosistémicos de cuencas hidrológicas y bosques (Menchaca y Alvarado, 2011).

También se ha investigado el factor antrópico en relación con la calidad del agua en relación a la contaminación de los compuestos químicos, metales pesados y organismos microbiológicos, así como las posibles enfermedades por el uso y consumo del agua de la microcuenca del río Pixquiac. Se ha encontrado que existen contaminantes que exceden los límites máximos permisibles de la Norma Oficial Mexicana NOM-127-SSA1-1994, "salud ambiental, agua para uso y consumo humano-límites permisibles de calidad y tratamientos a que debe someterse el agua para su potabilización", como son indicadores físico-químicos: fenoles, detergentes y cianuro; metales pesados: cadmio, plomo y arsénico; microbiológicos: coliformes fecales y coliformes totales (Menchaca, et al, 2015). Cabe señalar que, lo anterior se fundamenta en una base de datos del OABCC, que integra registros trimestrales de los parámetros de la calidad del agua en las presas de Medio Pixquiac, Socoyolapan y Cinco Palos, ubicadas en la microcuenca del río Pixquiac.

Cabe señalar, que como resultado de los anteriores estudios relacionados con el factor an- trópico y la calidad de agua en la microcuenca del río Pixquiac, se establece que los cuerpos de agua naturales están siendo impactados y afectados con niveles significativos de contaminación producidos por las diferentes actividades productivas y domésticas que se realizan, lo que compromete la disponibilidad del recurso hídrico para consumo humano.

Lo anterior, remite a la importancia de investigar los contaminantes que exceden los límites máximos permisibles como son fenoles, detergentes, cadmio, plomo, cianuro, arsénico, coliformes fecales y totales; así como identificar las actividades antrópicas que contribuyen a la contaminación del agua, y pueden incidir en la salud tanto humana como la de los ecosistemas de la región de estudio.

Los referentes establecidos, fueron la base para seleccionar la contaminación de cadmio como factor de estudio, debido a que es un metal pesado que tiene repercusiones relacionadas con la salud humana, ya que beber agua con niveles elevados, produce irritación grave del estómago, lo que causa vómitos, diarrea y en ciertas ocasiones la muerte.

Por otro lado, si se ingieren niveles de cadmio bajos durante periodos prolongados se produce acumulación en los riñones; además, el Departamento de Salud y Servicios Humanos (DHHS) y la Agencia Internacional para la Investigación del Cáncer (IARC), han determinado que el cadmio y los compuestos de cadmio son cancerígenos (ATSDR, 2012), cuestión que está directamente relacionada con la vulnerabilidad social de la población.

Con base en lo anterior, el problema de investigación tiene como eje conocer ¿cuáles son las actividades antrópicas de los usuarios de agua agrícola, pecuaria y doméstica que contribuyen a la contaminación del agua por cadmio en la microcuenca del río Pixquiac, Veracruz? Esta investigación se integra por las variables de factor antrópico entendido como las actividades de los usuarios del agua agrícola, pecuario y doméstico (variable independiente), que causan la contaminación de agua por cadmio (variable dependiente).

Se define al factor antrópico como todos los eventos provocados por las actividades huma- 
nas de los usuarios del agua, los que afectan a los ecosistemas y sus servicios ambientales, afectando a las fuentes naturales de agua, con cadmio; y a la contaminación de agua por cadmio, como la presencia o incorporación a los cuerpos de agua naturales de agentes químicos, sustancias o elementos tóxicos que contienen cadmio, que son perjudiciales para el hombre y/o para los ecosistemas (Menchaca, et al., 2015).

\section{Metodología}

\section{a. Zona de estudio}

La microcuenca del río Pixquiac tiene una extensión total de $106 \mathrm{~km} 2$ y se encuentra dentro de la cuenca del río La Antigua, en la región hidrológica del Papaloapan, Veracruz, México. Su corriente principal, el río Pixquiac, nace en la vertiente nororiental del sistema montañoso del Cofre de Perote, a una altura de 3,760 msnm y se une a los 1,030 msnm con el río Sordo. Además del río Pixquiac, en esta microcuenca escurren 212 ríos: 24 perennes y 188 intermitentes. El caudal de estos ríos se emplea principalmente en usos agropecuarios, industriales y público-urbanos (CONAGUA, 2010).

La vegetación predominante es el bosque mesófilo de montaña, bosque de pino-encino, bosque de coníferas y bosques secundarios de acahuales, cuya extensión abarca 70\% de la superficie total de la microcuenca; el 30\% restante de esta superficie se destina a las actividades agropecuarias y a los asentamientos humanos (García et al., 2008). Estos ecosistemas producen diversos beneficios y/o servicios ambientales tanto para las poblaciones locales como para la ciudad de Xalapa, los cuales están ligados a la disponibilidad y calidad del agua. Sin embargo, las actividades agropecuarias, domésticas, industriales, entre otras, así como la extracción ilegal de madera, carbón y leña amenazan los servicios ambientales. La preservación y gestión sustentable de este sistema natural debe constituir una prioridad para las autoridades gubernamentales del estado.

En 2010, el volumen de concesión fue 32.48 millones de m3 para los diversos usuarios de la microcuenca del río Pixquiac, siendo sus principales usos consuntivos el público-urbano (con un 68.64\% del caudal total), seguido por el uso industrial (22.07\%) y el agrícola (9.28\%) (CONAGUA, 2010). Esta región tiene una elevada disponibilidad de agua cuyo volumen asciende a 15,392.54 m3/año (García et al., 2008).

Sin embargo, la abundancia relativa de este recurso no se traduce en un mayor acceso para la población o una gestión más sustentable, debido a la contaminación de sus fuentes. Esta situación reduce el volumen disponible que puede consumirse de manera segura, además de incrementar los niveles de escasez del agua. Adicionalmente, no toda el agua que se extrae en la región es aprovechada por sus habitantes ya que la microcuenca del río Pixquiac es una de las principales fuentes de suministro para la ciudad de Xalapa y la región, que son tres presas que se abastecen de afluentes naturales que son: Medio Pixquiac, Socoyolapan y Cinco Palos (ver figura 1).

\section{b. Muestreo}

El número de cuestionarios aplicados a la población fueron de 42 para que la muestra fuera representativa, mismo que se determinó a través de muestreo aleatorio, con base en el número total de habitantes de las siete localidades de estudio, que es de 779. Se señala que, el criterio de selección se estableció de acuerdo a las personas que en las localidades de interés, desarrollaban las actividades de los sectores agrícola, pecuario y doméstico. Las localidades, pertenecientes a la microcuenca del río Pixquiac, se distribuyen de la siguiente manera: en la zona alta, se ubican las localidades del Ingenio del Rosario del municipio de Coatepec con una población de 228 habitantes y Cuesta del Vaquero con 40 habitantes del municipio de Acajete; en la zona media se encuentran Vega de Pixquiac con 42 habitantes, El Zapotal con una población de 143 personas, ambas se localizan en el municipio de Acajete; y en zona baja se ubica la localidad de Chilacayotes con 43 habitantes, Los Capulines con una población de 153 habitantes y El Tejocotal con un total de 130 personas, todas estas pertenecen a San Andrés Tlalnelhuayocan, (INEGI, 2010). 

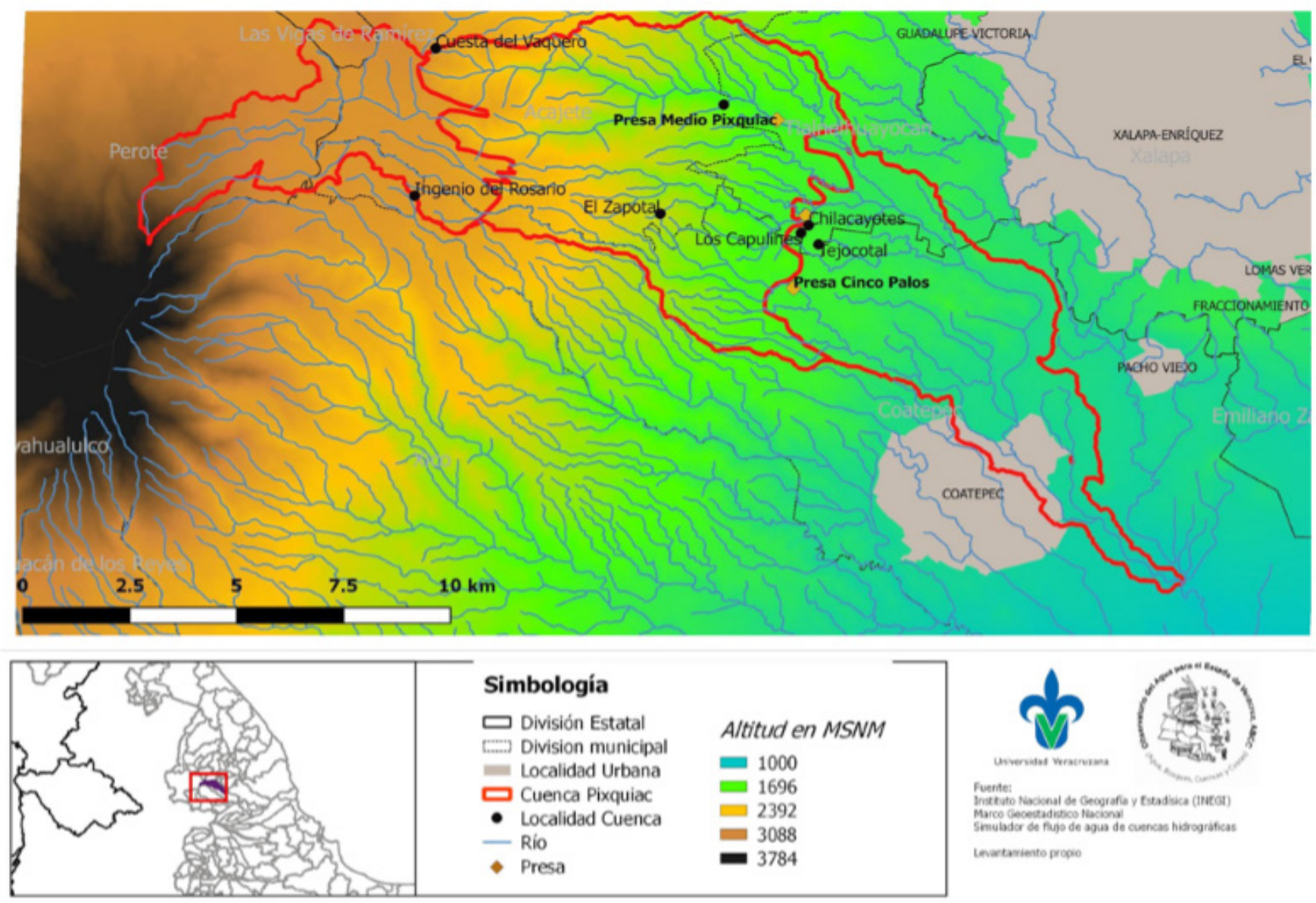

Se señala además que, con el propósito de identificar el factor antrópico que está impactando y afectando a la calidad del agua, la muestra de cada localidad (número de cuestionarios aplicados), se determinó con base en el tipo de muestreo no probabilístico de conveniencia, esto de acuerdo con los criterios de: cercanía en relación a los afluentes del agua en donde se realizan las actividades de los usuarios de interés; y por el flujo y/o escurrimiento de los cuerpos de agua que son afluentes a las presas de Medio Pixquiac, Socoyolapan y Cinco Palos, sitios donde se tienen los registros de la contaminación de agua por cadmio, donde se ubican dichas localidades.

\section{c. Procedimiento}

Se elaboró un mapa hidrológico y altimétrico de la microcuenca para el análisis del flujo de los cuerpos de agua superficiales, y para ubicar las localidades aledañas a las tres presas de estudio, donde se registró la contaminación del agua por cadmio; se diseñaron tres cuestiona- rios dirigidos a los usuarios del agua agrícola, pecuario y doméstico, para recabar información acerca de las actividades que se realizan y los productos que se usan cotidianamente. Cabe señalar, que previo a lo anterior, se identificaron las posibles actividades antrópicas que pudieran estar relacionadas con el cadmio, según la literatura científica.

En el trabajo de campo se identificó la distribución geográfica de las actividades agrícolas, ganaderas, y domésticas de los usuarios del agua, misma que no es homogénea en el territorio de la microcuenca de interés, ya que no en todas localidades se realizan las mismas actividades de los usuarios del agua.

Posteriormente se diseñaron y desarrollaron dos bases de datos relacionadas con la variable independiente y los indicadores del factor antrópico; y con la variable dependiente y los indicadores que es la contaminación del agua por cadmio.

El trabajo se realizó en un periodo de tres meses que abarcó desde diciembre de 2016 hasta 
febrero de 2017, lapso en el cual se visitaron las siete localidades de interés para aplicar los cuestionarios a los usuarios del agua agrícola, pecuario y doméstico.

\section{Resultados}

A. Actividades antrópicas de los usuarios del agua de los sectores agrícola, pecuario y doméstico que pueden contribuir a la contaminación de agua por cadmio.

\section{Usuarios del agua del sector agrícola}

Los principales cultivos en la zona de estudio son: maíz, policultivo de maíz y frijol, papa, y policultivo de maíz y papa, esto en las localidades de Los Capulines, Chilacayotes, Tejocotal, Ingenio del Rosario, Cuesta del Vaquero y Vega del Pixquiac, mismas que pertenecen a la microcuenca del río Pixquiac. La extensión aproximada de dichos cultivos, es de 49 hectáreas aproximadamente, mismas que están cercanas a las presas de Medio Pixquiac, Socoyolapan y Cinco Palos, sitios donde se tienen los registros de la calidad de agua. A continuación, se presentan los porcentajes que indican las actividades de los usuarios del agua en dicho sector, que contribuyen a la contaminación del agua por cadmio:

El mayor porcentaje de uso de agroquímicos es de herbicidas, ya que un 54\% de la población total los utiliza; en la zona de estudio se encontraron cuatro distintos tipos que son: Durmina 40, Rudo, Sencor y Surfacid, éstos pueden estar relacionados con la contaminación de cadmio (Cd), ya que en su composición contiene $\mathrm{Cd}$ en cantidades de 32.7 Hg.g-1, (Metrohm, 2017); la cantidad de herbicidas que se utiliza es de aproximadamente $22 \mathrm{~L}$ del concentrado, con una frecuencia de aplicación anual (ver figura 2).

El segundo agroquímico es el uso de insecticidas con un 46\% del 100\% de la población incluida en el estudio; el tipo de éstos agroquímicos que se usan en la zona de estudio es: Interfuran 350 y Monocrotofos; no se tiene las cantidades de concentración de cadmio de los compuestos; sin embargo, varios autores confirman que son fuentes antrópicas de cadmio (Rodríguez et al., 2008); la medida de herbicidas que se usa es de aproximadamente $21 \mathrm{~L}$ de concentrado, con una frecuencia de aplicación anual (ver figura 2).

El tercer lugar está el uso de fertilizantes orgánicos con un 38\% del total de usuarios del agua el sector agrícola entrevistados; siendo nueve diferentes tipos los que se aplican en la zona de estudio: Axefol K-45, Codacal boro, Macrofol, Kel-Cab, Poliquelmulti, Axifer 300, Fertimax 20-10-10, Maxi- grow Excel y K-Fol; su composición contiene $\mathrm{Cd}$ en cantidades que varían entre 8 y 500 mg kg-1 (Bonomelli et al., 2003); la cantidad de fertilizantes que se utiliza es más de 600 kg, y es aplicado con una frecuencia bimestral o anual (ver figura 2).

El cuarto lugar corresponde tanto al uso de raticidas como los fungicidas, ambos con un 31\% del 100\% de los usuarios agrícolas. Respecto a los raticidas, las marcas del mercado son de diversos tipos; el contenido de cadmio es variable según del formula del producto, pero distintos autores confirman que son fuentes antrópicas relacionadas con cadmio (Acevedo et al., 2005); la cantidad que se usa es variable y de forma permanente o intermitente, según se requiera (ver figura 2).

\section{Figura 2. Agroquímicos usados en la microcuenca del río Pixquiac}

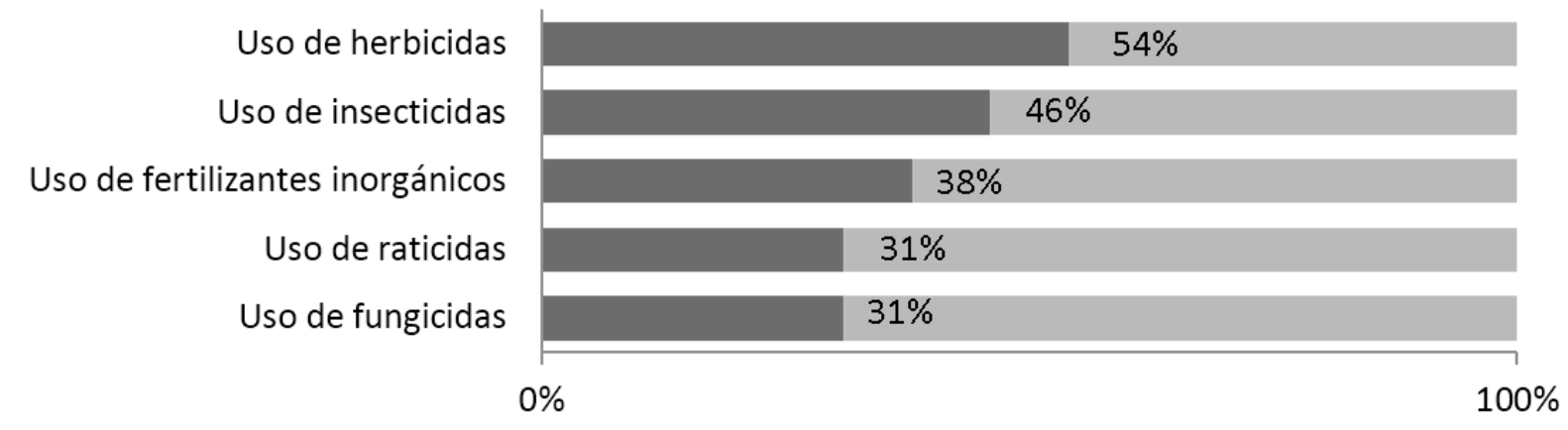


Respecto a los fungicidas, los distintos tipos de éstos productos son: Interguzan, Blanco 750, Dupont Curzate M-8, FLWAZ, Fungoxyl, Mancosol 80, Pulsor, Rolling 720; el contenido cadmio en la composición es de 1.535 mg Cd kg-1(Martí et al., 2009); la cantidad de fungicidas que se aplican es de aproximadamente $10 \mathrm{~L}$ de concentrado, con una frecuencia de aplicación anual (ver figura 2).

Cabe señalar que, el proceso de contaminación difusa ocurre cuando dichos compuestos químicos que contienen cadmio, se albergan en la tierra y transitan mediante procesos de precipitación hacia los cuerpos de agua naturales y/o las presas en donde se tiene la información sobre la calidad del agua.

\section{Usuarios de agua del sector pecuario}

En la microcuenca del río Pixquiac, los principales tipos de ganado que se crían son el ganado equino, bovino y el porcino en las localidades de Los Capulines, Chilacayotes, Tejocotal, Ingenio del Rosario, Cuesta del Vaquero y Vega del Pixquiac (ver figura 3).

Respecto a la contribución de cadmio por las actividades antrópicas pecuarias, se encontró que la producción de estiércol es considerada como una de las principales actividades productivas responsables de la contaminación difusa de los recursos hídricos (Alfaro y Salazar, 2005). Y también que, el cadmio llega mediante la aplicación de abono de estiércol (Pérez y Azcona, 2012). El agua es contaminada por excretas ganaderas directamente a través de escurrimientos, infiltraciones y percolación profunda en las granjas, e indirectamente por escorrentías y flujos superficiales desde zonas de pastoreo y tierras de cultivo (Pinos et al., 2012).

En la zona de estudio, la cantidad de estiércol que produce el ganado, según lo establecen los usuarios del agua del sector pecuario, es de 2000 kg (55\%); menos de 2000 kg (27\%); y más de 2000 kg (18\%), dicha generación de estiércol es en el periodo de un año. Lo anterior, establece que la producción de excretas puede estar contribuyendo a la contaminación de las fuentes de agua naturales de las presas arriba mencionadas (ver figura 4).

3. Usuarios del agua del sector doméstico en la microcuenca del río Pixquiac

Respecto a la posible aportación de cadmio de los usuarios de agua domésticos, se midie-

\section{Figura 3. Tipo de ganado en la microcuenca del río Pixquiac}

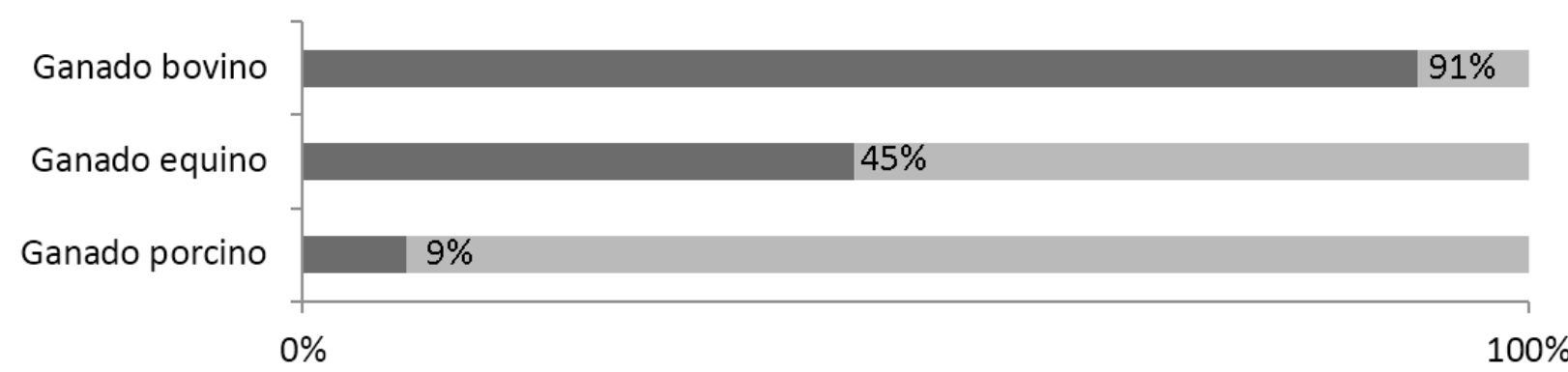

Figura 4. Producción de estiércol en la microcuenca del río Pixquiac

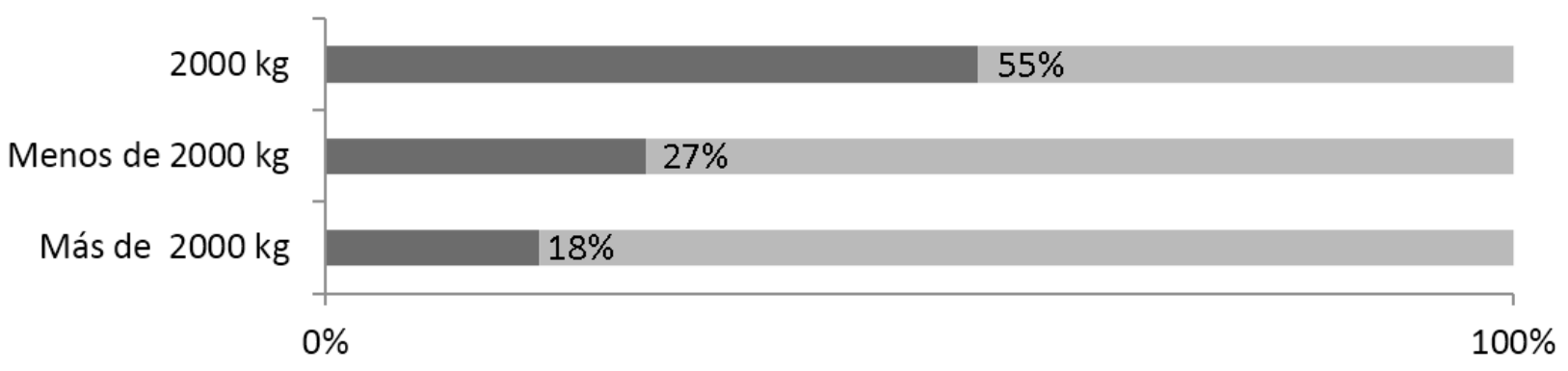


ron tres actividades antrópicas que se realizan en las localidades rurales estudiadas: uso de baterías y disposición final; quema de basura; así como uso de combustibles.

Respecto al uso de baterías, poco más de una cuarta parte de la población, esto es el 28\% de los habitantes de la región de estudio las utilizan, de éste el 40\% se entierra en la región de la microcuenca del río Pixquiac, recarga hidrológica importante, mientras que el 60\% los desechan con los demás residuos sólidos, los cuales son recolectados por el camión de basura, dándole una disposicón final fuera de la microcuenca. Se señala además, que las baterías níquel-cadmio (Ni-Cd), están compuestas en un 18\% de dicho elemento (Castro y Díaz, 2004). Cabe señalar que, si éstas no tienen una disposición final adecuada, y se entierran con los demás residuos sólidos, mediante la precipitación se forman lixiviados que se filtran llegando a las aguas subterráneas o se arrastra mediante la escorrentía contaminando las aguas superficiales (ver figura 5).
La quema de basura, es la segunda actividad antrópica doméstica que aporta cadmio, debido a que el 67\% de los habitantes de las localidades estudiadas incinera los residuos sólidos, los que por medio de la precipitación y escorrentía se arrastran a los cuerpos de agua superficiales de la región. Aunque no se tiene cuantificada la concentración de cadmio en esta actividad, distintos autores señalan que la incineración de residuos, como actividad antrópica, se relaciona con la contaminación del recurso hídrico por cadmio (Noriega et al., 2008) (ver figura 6).

La tercera actividad antrópica doméstica que contribuye con cadmio a la contaminación del agua es el uso de combustibles fósiles, ya que se incorpora al medio ambiente (Noriega et al., 2008). El 50\% de la población entrevistada, desarrolla ésta actividad; si bien, no se cuenta con la información precisa sobre la concentración de este elemento químico, que aporta el uso de combustibles fósiles, varios autores confirman que es una fuente de cadmio (Pérez y Azcona, 2012), (Martínez, et al., 2013) (ver figura 7).

Figura 5. Disposición final de las baterías en la microcuenca del río Pixquiac

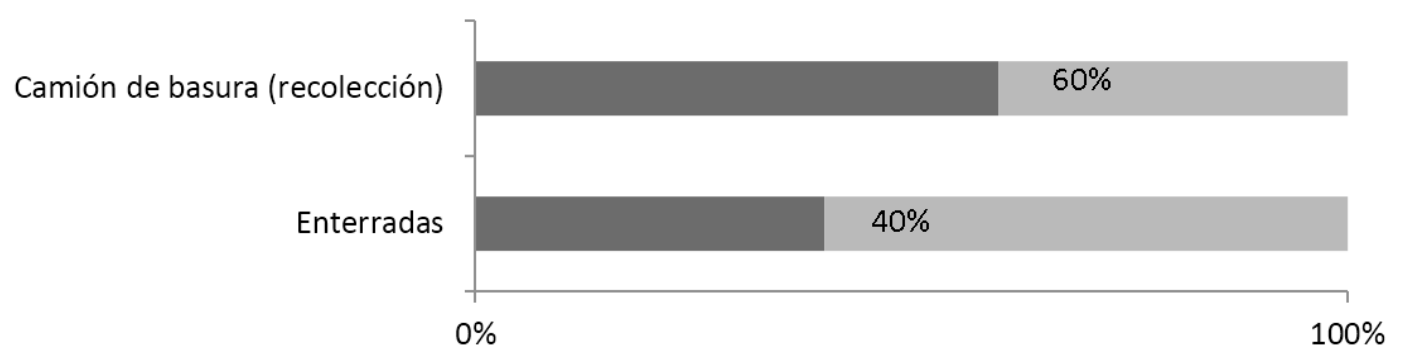

Figura 6. Incineración de residuos sólidos en la microcuenca del río Pixquiac

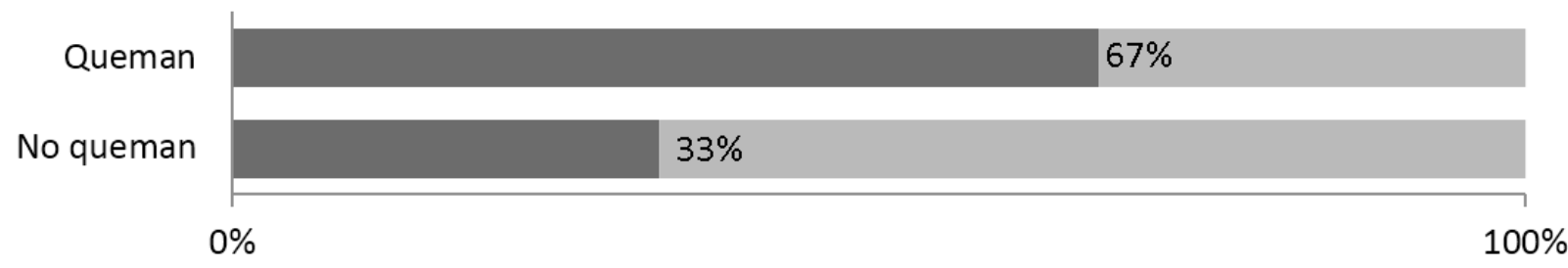

Figura 7. Uso de combustibles fósiles en la microcuenca del río Pixquiaci

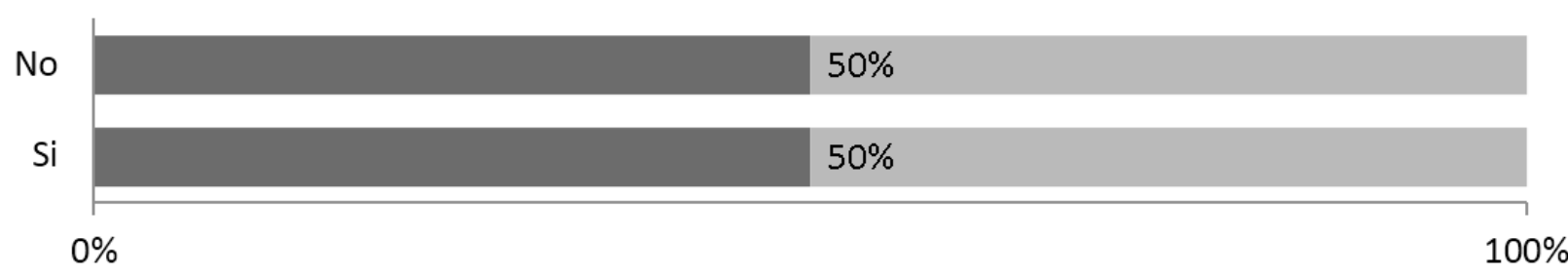




\section{B. Contaminación del agua por cadmio}

Respecto al comportamiento de la variable dependiente, los resultados muestran que las actividades antrópicas pueden estar impactando y afectando a los servicios ambientales conexos al agua, ya se observa que la concentración de cadmio en la microcuenca del río Pixquiac supera el límite máximo permisible (LMP) tanto de los valores de referencia de la Organización Mundial de la Salud (OMS) 1995, como el de la norma mexicana NOM-127-SSA1, (Salud ambiental, agua para uso y consumo humano-límites permisibles de calidad y tratamientos a que debe someterse el agua para su potabilización). Por otra parte, el límite máximo permisible de cadmio en la Norma Oficial Mexicana NOM-127-SSA1-1994 es de 0.005 $\mathrm{mg} / \mathrm{L}$, sin embargo, al revisar el promedio de las concentraciones de los indicadores de la calidad del agua del 2005-2016 en la microcuenca del río Pixquiac se observa que está por encima del LMP con una diferencia de 0.0019 mg/L. A su vez, si se compara con el promedio más una desviación estándar también supera el LMP establecido por la OMS con una diferencia de $0.0049 \mathrm{mg} / \mathrm{L}$. (ver tabla 1).

\section{Conclusiones}

Existen evidencias respecto a que distintas actividades antrópicas de los usuarios del agua de los sectores agrícola, pecuario y doméstico, están contribuyendo a la contaminación del agua por cadmio. Lo anterior, señala la relevancia de evaluar las actividades de carácter antrópico de los usuarios del agua, mismas que pueden estar relacionadas con la contaminación del agua. A partir de lo anterior, se pueden desarrollar metodologías de valoración del impacto y afectaciones antrópicas a los servicios ambientales ecosistémicos, en donde se incluyan un mayor número de contaminantes, mismos que pueden estar relacionados con otros, que están por arriba de los parámetros permitidos, en la microcuenca del río Pixquiac. Cabe señalar que, en el Observatorio del Agua el estado de Veracruz, actualmente se desarrollan, tanto dichas bases de datos, como las metodologías de valoración, lo que permite establecer los efectos que el hombre y/o la sociedad tienen en los ecosistemas conexos a dicho recurso natural.

\section{Referencias}

Acevedo, E., Carrasco, M., León, O., Martínez, E., Silva, P., Castillo, G., et al. (2005). Criterios de la calidad de suelo agrícola. Ministerio de Agricultura de Chile.

Alfaro, M. y Salazar, F. (2005). Ganadería y contaminación difusa, implicancias para el sur de Chile. Agricultura Técnica, 65(3), 330-340.

Agency for Toxic Substances and Disease Registry (ATSDR). (2012). Toxicological profile for cadmium. Washington D.C.

Bonomelli, C., Bonilla, C. y Valenzuela, A. (2003). Efecto de la fertilización fosfatada sobre el contenido de cadmio en cuatro suelos de Chile. Pesquisa Agropecuaria

Tabla 1. Comparación de los límites máximos permisibles de la NOM-127-SSA1-1994 y la concentración de cadmio en la microcuenca del río Pixquiac

\begin{tabular}{|c|c|c|c|c|}
\hline $\begin{array}{c}\text { Límite máxi- } \\
\text { mo permisible } \\
\begin{array}{c}\text { (mg/L) NOM-127- } \\
\text { SSA1-1994 }\end{array}\end{array}$ & $\begin{array}{c}\text { Promedio de las } \\
\text { concentraciones } \\
\text { de los indicadores } \\
\text { de la calidad del } \\
\text { agua 2005-2016 } \\
\text { de la microcuenca } \\
\text { (mg/L) }\end{array}$ & $\begin{array}{c}\text { Promedio más una } \\
\text { desviación están- } \\
\text { dar de los indicado- } \\
\text { res de la calidad del } \\
\text { agua en la micro- } \\
\text { cuenca (mg/L) }\end{array}$ & $\begin{array}{c}\text { Diferencia entre } \\
\text { LMP de la NOM- } \\
\text { 127-SSA1-1994 } \\
\text { y el promedio de } \\
\text { los indicadores de } \\
\text { la microcuenca } \\
\text { (mg/L) }\end{array}$ & $\begin{array}{c}\text { Diferencia entre } \\
\text { LMP de la NOM- } \\
\text { 127-SSA1-1994 y } \\
\text { el promedio más } \\
\text { una desviación } \\
\text { estándar de los } \\
\text { indicadores de } \\
\text { la microcuenca } \\
\text { (mg/L) }\end{array}$ \\
\hline 0.005 & $0.0069 a$ & 0.0099 & 0.0019 & 0.0049 \\
\hline
\end{tabular}

Fuente: Bases de datos del Sistema de Información del OABCC, 2017. 
Brasileira, 38(10), 1179-1186.

Castro, J. y Díaz, M. (2004). La contaminación por pilas y baterías en México. Gaceta ecológica, 72, 53-74.

Comisión Nacional del Agua (CONAGUA). (2010). Registro Público de Derechos del Agua (REPDA). México.

Evaluación de los ecosistemas del milenio (EM). 2005. Ecosistemas y bienestar humano: marco para la evaluación. World Resources Institute. 3-6 pp.

García, I., Martínez, A. y Vidriales, G. (2008). Balance hídrico de la cuenca del río Pixquiac. México, D.F.: Sendas.

Instituto Nacional de Estadística y Geografía (INEGI). (2010). XIII Censo de Población y Vivienda 2010. México.

Marsily, G. (2003). El agua. México, D.F.: Siglo Veintiuno.

Martí, L., Filippini, M., Bermejillo, A., Troilo, S., Salcedo, C. y Valdés, A. (2009). Monitoreo de cadmio y plomo en los principales fungicidas cúpricos comercializados en Mendoza, Argentina. Revista de la Facultad de Ciencias Agrarias, 41(2), 109-116.

Martínez, K., Souza, V., Bucio, L., Gómez, L. y Gutiérrez, M. (2013). Cadmio: efectos sobre la salud. Respuesta celular y molecular. Acta Toxicológica Argentina, 21(1), 3349.

Menchaca, S. y Alvarado, E. (2011). Efectos antropogénicos provocados por los usuarios del agua de la Microcuenca del río Pixquiac. Rev. Mexicana de C. Agrícolas, 1, 85-86.

Menchaca, S., Alvarado, E., Zapata, K. y Pérez, M. (2015). Construcción del riesgo por contaminación del agua y el principio de precaución. En Derecho y gestión del agua. México: Ubijus, 239-268.

Metrohm (2017). Cadmio y plomo en herbicida. Recuperado de https://www.metrohm.com/es-mx/aplicaciones/\%7B21F15A2E-224B-4E30-8866-BDDB9CEFOD56\%7D. Fecha de consulta 10 de diciembre de 2017.

Noriega, P., Medici, A., Murillo, A., Bedón, J., Haro, F. y Galecio, G. (2008). Estudio de la concentración de cadmio y plomo en el aire de la ciudad de Quito, empleando briofitas como biomontones. La granja, 8(2), 17-24.

Pérez, P. y Azcona, M. (2012). Los efectos de cadmio en la salud. Revista de especialidades Médico-Quirúrgicas, 17(3), 199-205.

Pinos, J., García, J., Peña, L., Rendón, J., Gónzalez, C. y Tristán, F. (2012). Impactos y regulaciones ambientales del estiércol generado por los sistemas ganaderos de algunos países de América. Agrociencias, 46 (4), 359-370.
Rodríguez-Serrano, M., Martínez-de la Casa, N., Romero-Puertas, M., del Río, L. y Sandalio, L. (2008). Toxicidad del cadmio en plantas. Ecosistemas, 17(3), 139-146.

Secretaría de Medio Ambiente y Recursos Naturales (SEMARNAT). (2004). Introducción a los servicios ambientales. México: Autor. 